\title{
Static-dynamic algorithm for managing asynchronous computations in distributed environments
}

\author{
S A Gorsky ${ }^{1}$ and A G Feoktistov ${ }^{1}$ \\ ${ }^{1}$ Matrosov Institute for System Dynamics and Control Theory of SB RAS, \\ Lermontov St. 134, Irkutsk, Russia, 664033 \\ gorsky@icc.ru
}

\begin{abstract}
The paper addresses a relevant problem of computation scheduling in scientific applications (distributed applied software packages) executed in distributed environments. Forming an optimal schedule of jobs for executing of applied software (modules) is an NP-hard problem. Therefore, in practice, heuristic methods of scheduling are often used. In this regard, we propose a new static-dynamic algorithm for managing computations in heterogeneous distributed environments. The results of operating the proposed algorithm are simulated in comparison with other scenarios for computing management. They show that applying the algorithm makes it possible to achieve a rational balance between the scheduling time and the computations makespan.
\end{abstract}

\section{Introduction}

Applications developed for supporting scientific workflows include a variety of software that implements data processing and analysis, different computational methods, and processes for modeling the systems under study. Typically, such software is based on a modular approach and oriented to highperformance computing [1]. Inputs and outputs of modules determine relations between them. In this case, the computational job describes executed module, its inputs and outputs, features of launching the module, and requirements to a computing environment. Thus, a workflow consists of a set of interconnected jobs. Additional job specification includes information about relations between parent and child jobs.

When a workflow is executed in a parallel or distributed computing environment of the public access, non-trivial problems arise in determining the order of workflow job executions on limited resources of the environment. In general, the formation of the optimal schedule of jobs is NP-hard [2].

We focus on asynchronous computations. Within the framework of such computations, workflow modules are launched when their input data is ready.

In the paper, we consider execution workflows under uncertainties in distributed applied software packages that relate to a special class of scalable scientific applications [3]. The presence of uncertainties determines the use of heuristic approaches to scheduling computations.

Such heuristics should rely on known relations between modules. In addition, considering data placement when executing workflow modules is also essential.

\section{Related work}

Developers of scientific applications distinguish between two types of workflows: abstract and concrete [4]. Jobs for executing workflows of both types consist of sets of interrelated sub-jobs describing 
executable programs (modules) and requirements (number of processors or cores, sizes of RAM and disk memory, etc.) to resources of a computing environment.

In the paper, we consider abstract workflows, jobs and data of which are not related to specific resources of computational environments. In general, such environments can integrate public access resources with additional resources from various grid and cloud platforms.

Well-known Workflow Management Systems (WMSs) implement both the resource allocation and jobs execution of abstract workflows on dedicated resources [5-7]. Often, an abstract workflow is presented in the form of a Directed Acyclic Graph (DAG), which reflects relations between modules or jobs of a workflow [8]. Therefore, many WMSs (for example, Pegasus [9]) use popular tools such as Condor DAGMan [10] or Gridway [11] that support managing similar relation description structures of computing tasks. Often, Condor DAGMan is independently applied for managing workflows.

Within the framework of our study, we assume the presence of uncertainties in the job execution time and the amount of transferred data. In this case, when selecting resources, such tools apply simple heuristics, such, for example, as the allocation of the first available node with suitable characteristics or the node with the least number of jobs [12].

Another popular approach is to split the workflow into a set of micro-workflows, each of which runs on a separate resource [13]. However, such a decomposition may not always be implementable for an arbitrary workflow owing to the possible lack of the necessary structural relations between workflow jobs [14].

In this regard, we propose a new heuristic, which in some cases allows us to improve the allocation of resources by taking into account the relations between jobs in comparison with the Condor DAGMan scheduler. When using this heuristic, the computation planning time is close to the time spent by the compared tool.

\section{Static-dynamic algorithm}

We have developed an algorithm that includes static and dynamic stages of operation. In the static stage, we assign priorities to workflow jobs according to our proposed heuristic. These priorities can then change dynamically in accordance with the data readiness for the execution of modules.

\section{A. Model Workflow}

As an example, let us consider the workflow represented in Figure 1 by a bipartite DAG, which includes two disjoint sets of vertices (a set of the parameters $d_{1}, d_{2}, \ldots, d_{11}$ and a set of the modules $\left.m_{1}, m_{2}, \ldots, m_{10}\right)$. Arcs reflect the data transfer between modules. Each $i$ th module has a one-to-one correspondence with the $i$ th job.

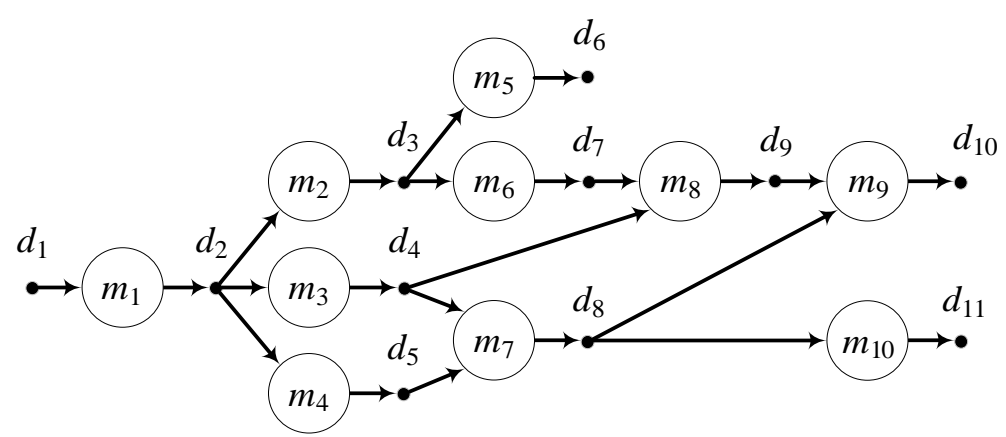

Figure 1. Workflow.

The initial data are represented by the parameter $d_{1} . d_{6}, d_{10}$, and $d_{11}$ are target parameters, whose values are to be calculated. We can see that the workflow is weakly structured in terms of the decomposition into a set of micro-workflows. 


\section{B. Heuristic}

The proposed heuristic is based on the analysis of causal relations between modules in the static stage of the algorithm operation. The execution of each module causes the further execution of a certain number of modules and the implementation of a certain number of parameter transfers. The analysis is carried out in order to determine the priorities of jobs for the execution of modules.

Assignment of jobs priorities begins with modules that no other module depends on. We apply a lexicographical rule for multi-criteria assigning the job priorities using the following four characteristics with their optimality conditions [15]:

- Number $n_{a} \rightarrow$ max of modules launch in the future caused by the module execution,

- Number $n_{t d} \rightarrow \max$ of parameters, whose transfers in the future are caused by the module execution,

- Sum $n_{p} \rightarrow$ max of priorities of modules, whose launches in the future are caused by the module execution,

- Number $n_{r d} \rightarrow \min$ of parameters that are received by a module.

In comparison with the lexicographical method mentioned in [15], we use estimates for values of characteristics instead of the values themselves. To obtain such estimates, we apply the original algorithm [16].

The results of assigning priorities to the jobs of the workflow shown in Figure 1 are represented in Table I. The lexicographical rule applied three times to the following groups of modules: $\left\{m_{5}, m_{9}, m_{10}\right\}$, $\left\{m_{6}, m_{7}\right\}$, and $\left\{m_{2}, m_{3}\right\}$.

Table 1. Priorities of jobs.

\begin{tabular}{ccccccc}
\hline Module & $n_{a}$ & $n_{t d}$ & $n_{p}$ & $n_{r d}$ & Job & Job priority \\
\hline$m_{1}$ & 9 & 12 & 45 & 1 & $j_{1}$ & 10 \\
$m_{2}$ & 4 & 4 & 14 & 1 & $j_{2}$ & 9 \\
$m_{3}$ & 4 & 4 & 12 & 1 & $j_{3}$ & 8 \\
$m_{4}$ & 3 & 3 & 8 & 1 & $j_{4}$ & 7 \\
$m_{5}$ & 0 & 0 & 0 & 2 & $j_{5}$ & 3 \\
$m_{6}$ & 2 & 2 & 5 & 1 & $j_{6}$ & 6 \\
$m_{7}$ & 2 & 2 & 3 & 2 & $j_{7}$ & 5 \\
$m_{8}$ & 1 & 1 & 1 & 2 & $j_{8}$ & 4 \\
$m_{9}$ & 0 & 0 & 0 & 7 & $j_{9}$ & 1 \\
$m_{10}$ & 0 & 0 & 0 & 4 & $j_{10}$ & 2 \\
\hline
\end{tabular}

\section{Algorithms for Dynamic Stage}

The general scheme for job scheduling is represented by the main algorithm, which is realized using the function JobScheduling(). This scheme includes the following operations:

- Finding available resources that can be allocated,

- Determining jobs ready to be run, whose inputs have already been calculated,

- Resources allocation for the ready jobs,

- Preparing data transfer for allocated resources.

These operations are correspondingly implemented by the functions ResourceMonitoring(), JobMonitoring(), ResourceAllocation(), and DataTransferPreparation(). In addition, we use auxiliary functions GetDedicatedResources(), ResourseAvailable(), JobReady(), CheckPriority(), 
PrioritiesChanging(), ReadyJobAllocation(), and JobAllocationPrediction() which are not discussed in detail in the paper.

The simplified algorithms 1-5 for the key functions for monitoring resources and jobs, data transfer preparation, resource allocation, and job scheduling are given bellow. A monitoring system is represented in [16]. Data transferring preparation for modules is carried out only when they are guaranteed to be launched on the allocated resources in accordance with the priorities of their jobs.

Variables used in the aforementioned algorithms have the following interpretation:

- DAG $w f$,

- Integer vector $r=\left(r_{1}, r_{2}, \ldots, r_{k}\right)$ of dedicated resources, where $k$ is a number of resources,

- Boolean vector $v=\left(v_{1}, v_{2}, \ldots, v_{k}\right)$ of resource states, where $v_{i}=1\left(v_{i}=0\right)$ shows that the $i$ th resource is available (not available),

- Boolean vector $\mathrm{w}=\left(w_{1}, w_{2}, \ldots, w_{l}\right)$ of job states, where $w_{i}=1\left(w_{i}=0\right)$ shows that the $i$ th job is ready (not ready),

- Real vector $p=\left(p_{1}, p_{2}, \ldots, p_{l}\right)$ of job priorities,

- Boolean matrix $A$ of the dimensions $l \times k$ shows the result of resource allocation for the ready jobs, where $a_{i j}=1\left(a_{i j}=0\right)$ means that the $j$ th resource is allocated (not allocated) to the $i$ th resource.

- Boolean matrix $X$ of the dimensions $m \times k$ defines the placement of parameters on resources, where $x_{i j}=1\left(x_{i j}=0\right)$ shows that the $i$ th parameter is placed (not placed) on the $j$ th resource, where $m$ is a number of parameters.

- Boolean matrix $Y$ of the dimensions $m \times k$ reflects parameter transfers, where $y_{i j}=1\left(y_{i j}=0\right)$ shows that the $i$ th parameter will be transferred (will not be transferred) to the $j$ th resource.

The algorithms considered above form the basis of the proposed static-dynamic algorithm. This algorithm is implemented in the Orlando Tools framework used to develop distributed applied software packages [17].

\begin{tabular}{|c|c|}
\hline & \\
\hline 1 function ResourceMonitoring() & \begin{tabular}{|l|} 
Algorithm 2 for job monitoring \\
1 function JobMonitoring()
\end{tabular} \\
\hline 2 // Getting a list of dedicated resources & 2 // Initializing job states \\
\hline$r \leftarrow$ GetDedicatedResources() & $3 \quad w \leftarrow(0,0, \ldots, 0)$ \\
\hline 4 // Initializing resource states & 4 // Determining the job states (ready or not \\
\hline$v \leftarrow(0,0, \ldots, 0)$ & ready) \\
\hline 6 // Determining the current state of a resource & for $i=1 . . l \mathrm{do}$ \\
\hline for $i=1 . . k$ do & if $\operatorname{JobReady}(i)=1$ then \\
\hline if ResourseAvailable $\left(r_{i}\right)=1$ then & $w_{i}=1$ \\
\hline$v_{i}=1$ & end if \\
\hline else & end do \\
\hline$v_{i}=0$ & $10 \quad$ return $w$ \\
\hline 12 end if & end function \\
\hline \multicolumn{2}{|l|}{$\begin{array}{ll}13 & \text { end do } \\
14 & \text { return } v\end{array}$} \\
\hline 14 return $v$ & \\
\hline \multicolumn{2}{|l|}{15 end function } \\
\hline
\end{tabular}




\begin{tabular}{|c|c|c|}
\hline \multicolumn{2}{|r|}{ Algorithm 3 for data transferring preparation } & Algorithm 4 for resource allocation \\
\hline 1 & function DataTransferPreparation $(A, X)$ & 1 function ResourceAllocation $(w f, v, w, p, X)$ \\
\hline 2 & // Initializing data transferring & 2 // Initializing resources allocation \\
\hline 3 & $Y \leftarrow(0,0, \ldots, 0 ; 0,0, \ldots, 0 ; \ldots ; 0,0, \ldots, 0)$ & $A \leftarrow(0,0, \ldots, 0 ; 0,0, \ldots, 0 ; \ldots ; 0,0, \ldots, 0)$ \\
\hline 4 & for $i=1 . . l$ do & 4 // If the priority of the ready job is less than \\
\hline 5 & for $j=1 . . k$ do & 5 // the priorities of the unready jobs, \\
\hline 6 & $\begin{array}{l}\text { // Analyzing the } j \text { th resource allocation for the } \\
i \text { th module }\end{array}$ & 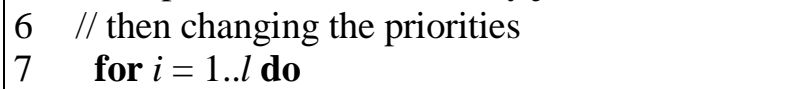 \\
\hline 7 & if $a_{i j}=1$ then & 8 if CheckPriority $(w f, i, p)$ then \\
\hline 8 & // Getting inputs of the $i$ th module & $p \leftarrow$ PrioritiesChanging $(w, p)$ \\
\hline 9 & $b \leftarrow \operatorname{GetInputs}(i)$ & end if \\
\hline 10 & for $q=1 . . m \mathrm{do}$ & 11 end do \\
\hline 11 & $\begin{array}{l}\text { // Checking the } q \text { th parameter placement on } \\
\text { the } j \text { th resource }\end{array}$ & $\begin{array}{l}12 \text { // Allocation of the ready jobs } \\
13 \quad A \leftarrow \text { ReadyJobAllocation }(w f, v, w, p, A)\end{array}$ \\
\hline 12 & if $b_{q}=1 \wedge x_{q j}=0$ then & 14 // Predicting the allocation for the unready jobs \\
\hline 13 & $\begin{array}{l}\text { // Preparing the } q \text { th parameter transfer on the } \\
j \text { th resource }\end{array}$ & $\begin{array}{l}15 / / \text { taking into account the job priorities and } \\
16 \text { // data placement }\end{array}$ \\
\hline 14 & $y_{q i}=1$ & $17 \quad A \leftarrow$ JobAllocationPrediction $(w f, v, w, p, A, X)$ \\
\hline 15 & end if & 18 return $(p, A)$ \\
\hline 16 & end do & 19 end function \\
\hline 17 & end if & \\
\hline & end do & \\
\hline & end do & \\
\hline 20 & return $Y$ & \\
\hline 21 & end function & \\
\hline
\end{tabular}

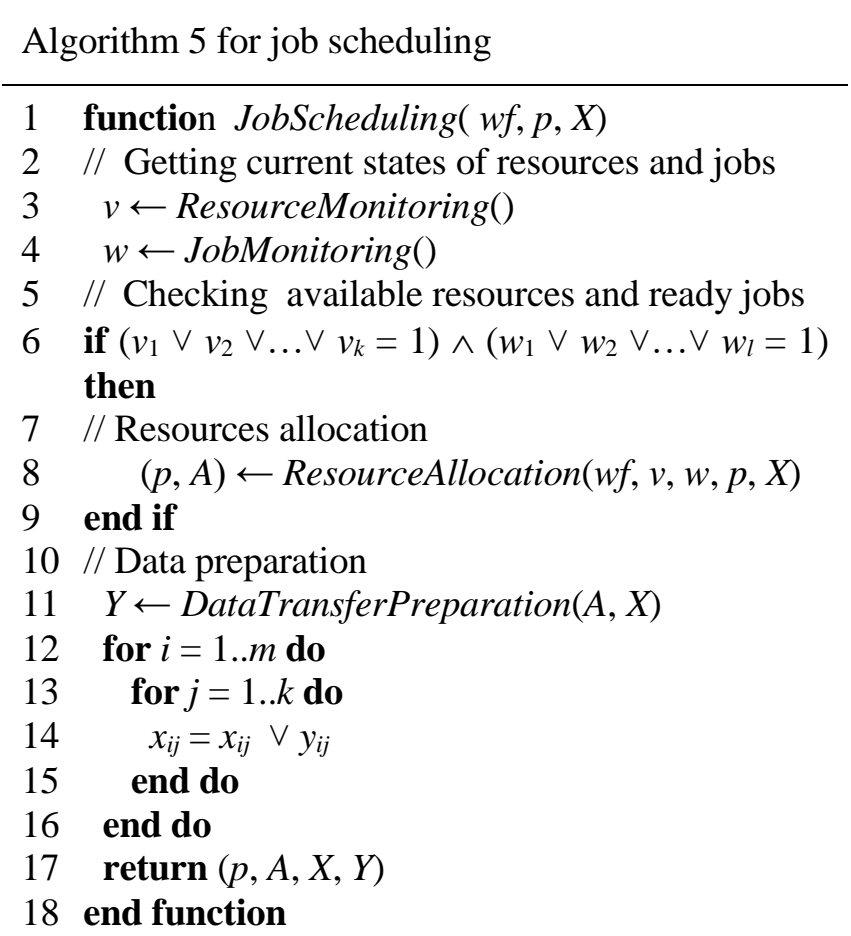




\section{Experiment}

In the first example, let us suppose that we have to execute flow of workflows in a computing environment consists of a set of Virtual Machines (VMs). Two VM with one core is allocated to running one workflow. All workflows have the structure shown in Figure 1.

This job flow implements parameters sweep computations [18]. Each job is executed with a unique variant of the source data. Therefore, the size of the data, their transfer time, and the execution time may differ for different jobs.

To demonstrate the result in operating our algorithms, we will consider a simple example with model time in applying to one workflow. Let there be the following execution times of jobs $j_{1}, j_{2}, \ldots, j_{10}: t_{1}=2$, $t_{2}=2, t_{3}=3, t_{4}=2, t_{5}=1, t_{6}=2, t_{7}=2, t_{8}=5, t_{9}=2$, and $t_{10}=1$. The transferring time for any data $\left(d_{1}\right.$, $\left.d_{2}, \ldots, d_{11}\right)$ is assumed to be equal to 1 . These times are proportional to the average computation results when solving a real problem in the public access Irkutsk Supercomputer Center [19].

Figure 2 and Figure 3 show the job scheduling by the Condor DAGMan algorithm and proposed static-dynamic algorithm. Optimal workflow makespan is equalled to 16 . The computation time with Condor DAGMan is 19. The static-dynamic algorithm reduces this time to 17 . This is a significant advantage for such an example.

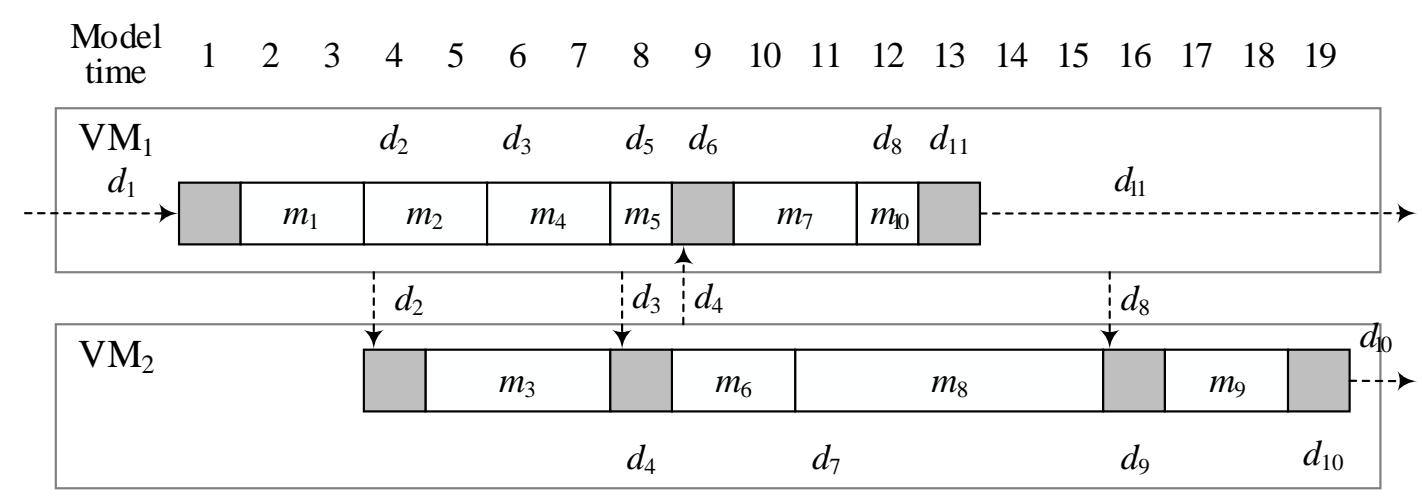

- Waiting for data

- Data transfer

Figure 2. Job scheduling via the Condor DAGMan algorithm.

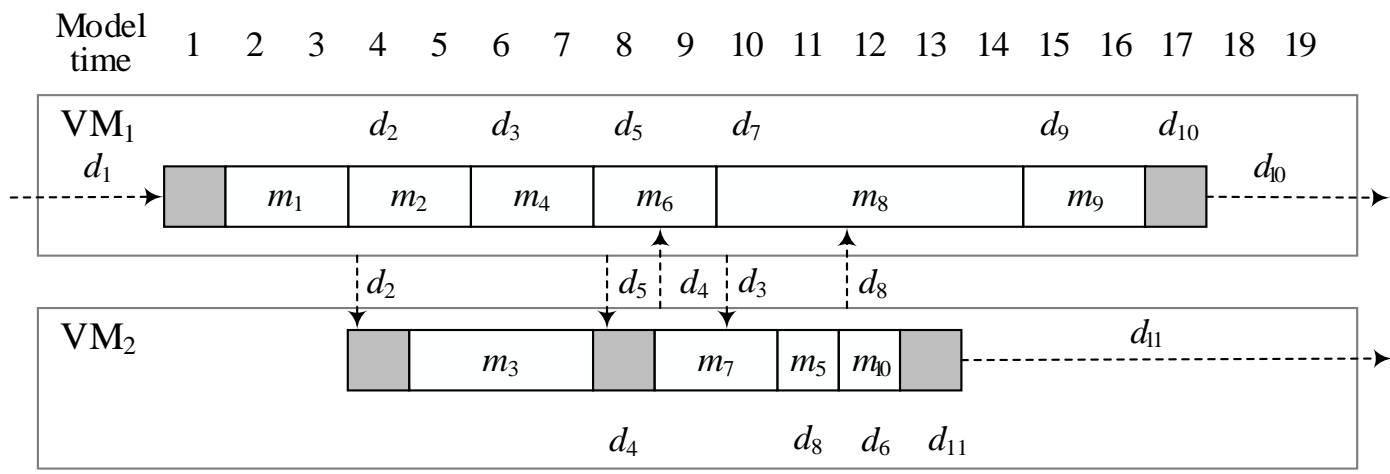

- Waiting for data

- Data transfer

Figure 3. Job scheduling via the proposed algorithm. 
We can see that both algorithms start out the same way. The $m_{1}$ modules $m_{1}, m_{2}$, and $m_{4}$ are consistently launched on $\mathrm{VM}_{1}$ in accordance with inter-module relations. In parallel, data is being prepared on $\mathrm{VM}_{2}$ for executing the module $m_{3}$.

After that, the operation of the algorithms begins to differ. The Condor DAGMan algorithm launches the $m_{5}$ module for execution on $\mathrm{VM}_{1}$. At the same time, our algorithm selects the $\mathrm{m}_{6}$ module.

According to the heuristic used by our algorithm, module $\mathrm{m}_{6}$ has a higher priority than module $m_{5}$. Really, more number of modules depends on the $m_{6}$ module in further computing within the workflow compared to the $m_{5}$ module.

Owing to launching the module $m_{5}$, the Condor DAGMan algorithm is forced to delay the execution of the $m_{6}$ module because of the need in preparing its input data on $\mathrm{VM}_{2}$.

In the given example, the reduction of the workflow runtime within our algorithm is also due to the preliminary transfer of the input data for the modules $m_{8}$ and $m_{9}$. Figures 4 and 5 show these transfers at the corresponding times.

The transfers of the parameters $d_{4}$ and $d_{8}$ are performed although the modules related to them are not yet ready to run. The parameter $d_{4}$ for the module $m_{8}$ is transmitted while the module $m_{6}$ is being executed (Figure 4). This is due to the fact that the module $m_{8}$ will be launched on the VM where the module $m_{6}$ is executing.

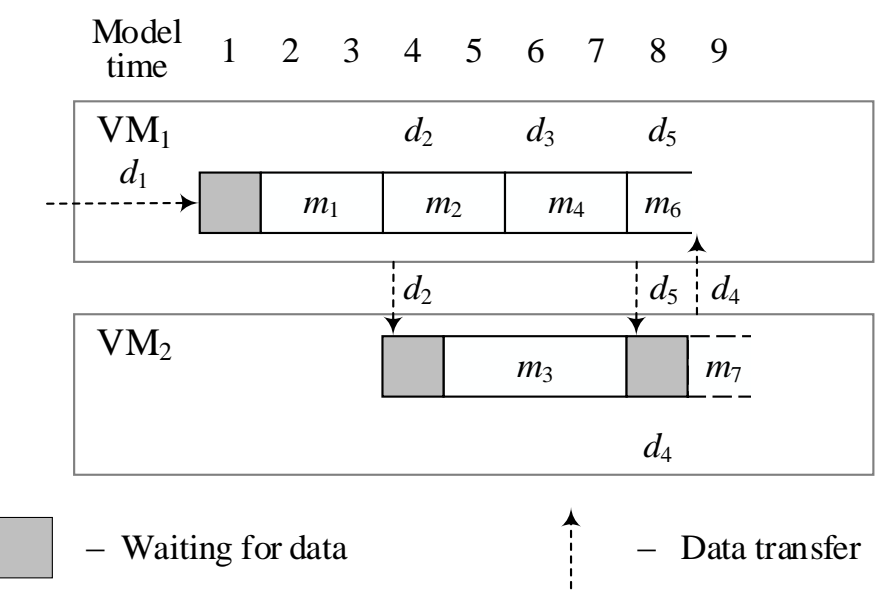

Figure 4. Preliminary data transfer 1.

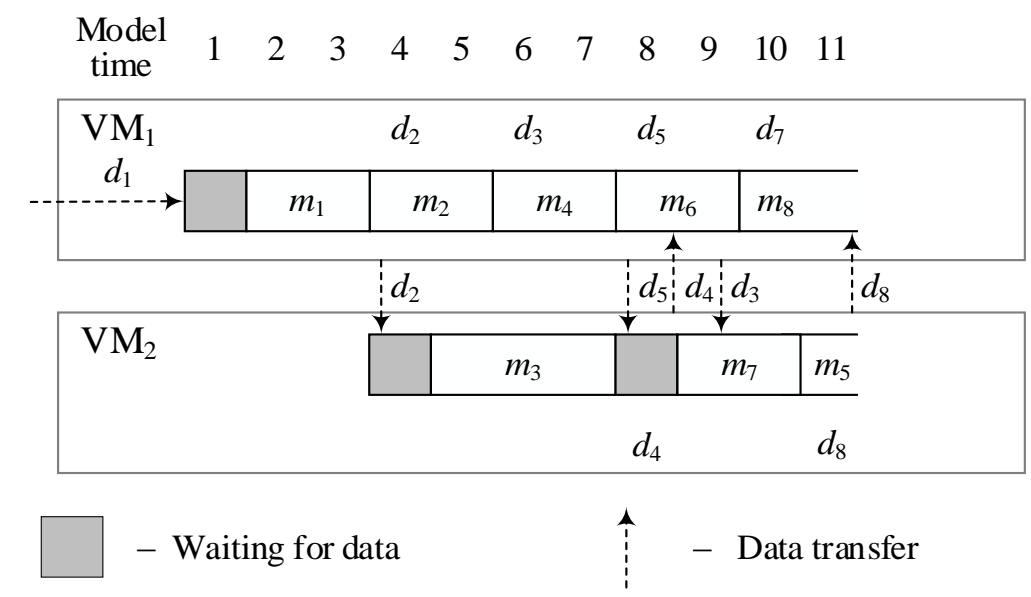

Figure 5. Preliminary data transfer 2 . 
The decision to transfer the parameter $d_{8}$ (Figure 5) is also obvious since the decision to launch the $m_{10}$ module has already been made. In this case, it remains to run the $m_{9}$ module. The module $m_{8}$ will calculate the parameter $d_{9}$ required for the module $m_{9}$.

Let us consider the issue of selecting a module for its launch on a resource that is being freed. We have implemented the JobAllocationPrediction() function, which selects the module to run on the resource when it becomes free. This check is performed for all resources on which workflow modules are running. Within the check, a set of modules including modules that are not ready to run can exist (for example, by time 8 in Figure 5).

The JobAllocationPrediction() function checks how the completion of the module executing and resource freeing will affect the readiness of the remaining unexecuted modules. Moreover, it allows us to assign modules to resources that are not yet ready for launch. This assignment of modules to resources allows us to prepare the necessary input data to the time of their launch.

Within the considered example, there is another background data transfer. The parameter $d_{3}$ is transferred to the module $m_{5}$. However, this transfer does not affect the workflow runtime.

In the theory, the preparation of the input data for the modules could be performed through overabundant sending the calculated parameters to all available computing resources. However, this solution has significant drawbacks. In practice, data transfers can degrade compute performance and slow down other transfers of the current data. Typically, the negative impact of data over-sending increases with the number of resources used and the number of modules executed. Thus, the overabundant data sending is highly undesirable for scalable workflows focused on a large number of resources.

For the job flow in the whole, the proposed algorithm reduces the total computation time by more than $11 \%$, taking into account the change in the module execution times in different jobs. At the same time, the time spent on job scheduling by the proposed algorithm does not exceed the time of the Condor DAGMan operating.

The next example addresses a workflow with the parallel list of data (Figure 6). This is an important feature of the workflow.

Let there be the following execution times of jobs $j_{1}, j_{2}$, and $j_{3}$ for executing the modules $m_{1}, m_{2}$, and $m_{3}: t_{1}=1, t_{2}=2$, and $t_{3}=1$. The transferring time for any data $\left(d_{1}, d_{2}, d_{3}, d_{4}\right)$ in Figure 6 is assumed to be equal to 1 .

$d_{2}$ and $d_{3}$ are the parallel lists of data. Each element $d_{2 i}$ of the parallel list $d_{2}$ is processed by the $i$ th instance of the module $m_{2}$ (Figure 70. The result of the execution of $m_{2 i}$ is saved in the element $d_{3 i}$ of the parallel data list $d_{3}$.

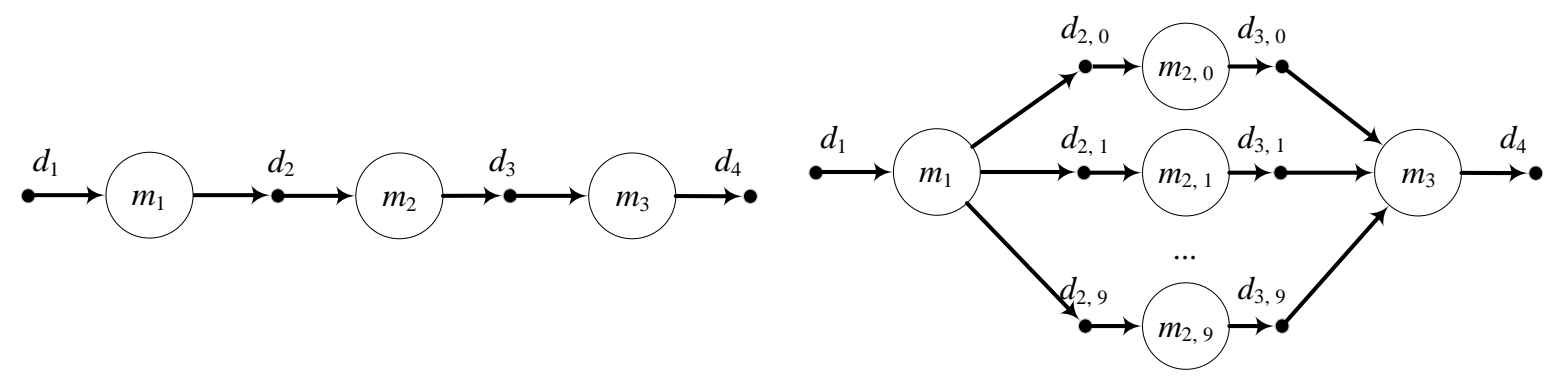

Figure 6. Initial workflow.

Figure 7. Workflow with parallel list of data.

Figures 8 and 9 show the simulation of the considered algorithms for this example. As in the first example, the presence of tools for assigning preliminary data transfer allows us to reduce the overheads associated with sending input data for modules. In this example, the proposed algorithm provides the reduction by $13 \%$ in the workflow makespan. 


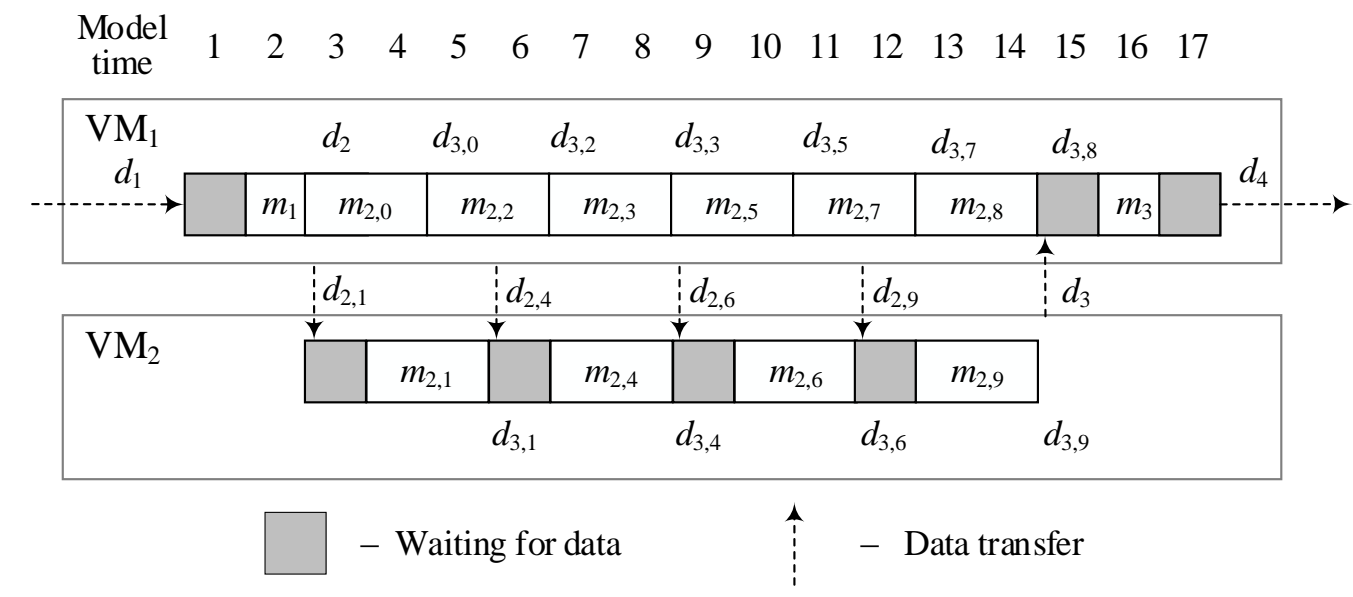

Figure 8. Job scheduling via the Condor DAGMan algorithm for workflow with parallel list of data.

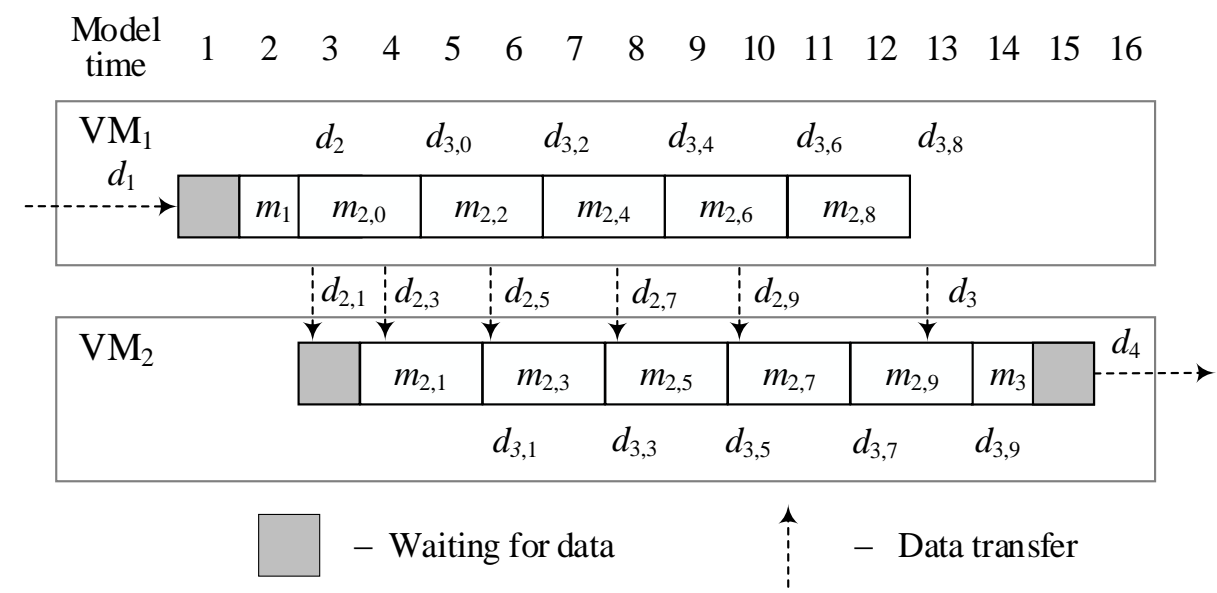

Figure 9. Job scheduling via the proposed algorithm for workflow with parallel list of data.

\section{Conclusions}

In the paper, we present a new algorithm that implements static-dynamic scheduling asynchronous computations in distributed applied software packages. The algorithm operation is oriented to heterogeneous distributed environments.

Through the experimental results, we demonstrated that the advantages of the proposed algorithm for different workflow types. In conditions of uncertainty over the modules execution time, the algorithm makes it possible to rationally determine the order of modules' launches in comparison with other ways of scheduling.

The advantages of the proposed algorithm for managing asynchronous computations in distributed environments have been demonstrated in specific examples. In the future, we hope to find theoretical estimates supporting such advantages for a larger number of cases.

As additional future work, we plan to use additional heuristics based on analyzing the modules execution time with test data in environment nodes within the framework of continuous integration, delivery, and deployment of package software.

\section{Acknowledgments}

The study was supported by the Ministry of Science and Higher Education of the Russian Federation, project no. 121032400051-9 «Technologies for the development and analysis of subject-oriented intelligent group control systems in non-deterministic distributed environments». 


\section{References}

[1] Hilman M H, Rodriguez M A and Buyya R 2020 Multiple workflows scheduling in multi-tenant distributed systems: A taxonomy and future directions ACM Comput. Surv. 53(1) 1-39

[2] Garey M and Johnson D 1979 Computers and Intractability (San Francisco: W. H. Freeman) p 338

[3] Feoktistov A, Kostromin R, Sidorov I and Gorsky S 2018 Development of distributed subjectoriented applications for cloud computing through the integration of conceptual and modular programming Proc. of the 41 st International Convention on information and communication technology, electronics and microelectronics (MIPRO-2018) (Riejka: IEEE) pp 256-261

[4] Atkinson M, Gesing S, Montagnat J and Taylor I 2017 Scientific workflows: Past, present and future Future. Gener. Comp. Sy.75 216-227

[5] Cao J, Jarvis S A, Saini S and Nudd G R 2003 Gridflow: Workflow management for grid computing Proc. of the 3rd IEEE/ACM International Symposium on Cluster Computing and the Grid (CCGrid 2003) (IEEE Press) pp 198-205

[6] Guler A T and Waaijer C JF, Palmblad M 2016 Scientific workflows for bibliometrics Scientometrics 107(2) 385-398

[7] Monge D and Garino C G 2010 Improving Workflows Execution on DAGMan by a Performancedriven Scheduling Tool Proc.of the 3rd Symposium on High-Performance Computing in Latin America 39 pp 3271-3285

[8] Tchernykh A, Feoktistov A, Gorsky S, Sidorov I, Kostromin R, Bychkov I, Basharina O, Alexandrov A and Rivera-Rodriguez R 2019 Orlando Tools: Development, Training, and Use of Scalable Applications in Heterogeneous Distributed Computing Environments Comm. Com. Inf. Sc. 979 265-279

[9] Deelman E, Vahi K, Rynge M, Juve G, Mayani R and Silva R F 2016 Pegasus in the cloud: Science automation through workflow technologies IEEE Internet Computing 20(1) 70-76

[10] Directed Acyclic Graph Manager. Available at: https://research.cs.wisc.edu/htcondor/dagman/dagman.html (accessed: 20.11.2020)

[11] Carrión IM, Huedo E and Llorente IM 2015 Interoperating grid infrastructures with the GridWay metascheduler Concurr. Comp.-Pract. E. 27(9) 2278-2290

[12] Kalayci S, Dasgupta G, Fong I, Ezenwoye O and Sadjadi S M 2010 Distributed and adaptive execution of Condor DAGMan workflows Proc. of the 22nd International Conference on Software Engineering and Knowledge Engineering pp 587-590

[13] Radchenko G, Alaasam A and Tchernykh A 2018 Micro-workflows: Kafka and kepler fusion to support digital twins of industrial processes Proc. of the 2018 IEEE/ACM International Conference on Utility and Cloud Computing Companion (UCC Companion) (IEEE Press) pp 83-88

[14] Bharathi S, Chervenak A, Deelman E, Mehta G, Su M-H and Vahi K 2008 Characterization of scientific workflows Proc. of the 3rd IEEE Workshop on Workflows in Support of Large-Scale Science (IEEE Press) pp 1-10

[15] Ho W, Xu X and Dey P K 2010 Multi-criteria decision making approaches for supplier evaluation and selection: A literature review Eur. J. Oper. Res. 202(1) 16-24

[16] Bychkov I V, Oparin G A, Feoktistov A G, Sidorov I A, Bogdanova V G and Gorsky S A 2016 Multiagent control of computational systems on the basis of meta-monitoring and imitational simulation Optoelectron. Instrum. Data Proces. 52(2) 107-112

[17] Feoktistov A, Gorsky S, Sidorov I, Bychkov I, Tchernykh A and Edelev A 2020 Collaborative development and use of scientific applications in Orlando Tools: Integration, delivery, and deployment Comm. Com. Inf. Sc.1087 18-32

[18] Buyya R, Murshed M, Abramson D and Venugopal S 2005 Scheduling parameter sweep applications on global Grids: a deadline and budget constrained cost-time optimization algorithm Software Pract. Exper. 35(5) 491-512

[19] Irkutsk Supercomputer Center. Available at: http://hpc.icc.ru (accessed: 10.05.2020) 